Check for updates

Cite this: Chem. Sci., 2019, 10, 1419

๑ All publication charges for this article have been paid for by the Royal Society of Chemistry

Received 20th September 2018 Accepted 14th November 2018

DOI: $10.1039 / c 8 s c 04188 a$

rsc.li/chemical-science

\title{
Catalytic farming: reaction rotation extends catalyst performance $\dagger$
}

\author{
Ayda Elhage, (D) Anabel E. Lanterna (D) and Juan C. Scaiano (iD *
}

The use of heterogeneous catalysis has key advantages compared to its homogeneous counterpart, such as easy catalyst separation and reusability. However, one of the main challenges is to ensure good performance after the first catalytic cycles. Active catalytic species can be inactivated during the catalytic process leading to reduced catalytic efficiency, and with that loss of the advantages of heterogeneous catalysis. Here we present an innovative approach in order to extend the catalyst lifetime based on the crop rotation system used in agriculture. The catalyst of choice to illustrate this strategy, $\mathrm{PdaTiO}$, is used in alternating different catalytic reactions, which reactivate the catalyst surface, thus extending the reusability of the material, and preserving its selectivity and efficiency. As a proof of concept, different organic reactions were selected and catalyzed by the same catalytic material during target molecule rotation.

\section{Introduction}

The industrial production of fine chemicals has led to the development of a plethora of different catalysts, many based on Pd complexes. ${ }^{1}$ Although many industrial applications utilize homogeneous Pd complexes, heterogeneous Pd metal-based systems are finding their place among industrial processes. The advantages of heterogeneous systems over their homogeneous counterparts are related to the ease of separation and reusability of the materials. ${ }^{2}$ Further, an increased interest in improved understanding of the catalytic activity of heterogeneous materials has led to exciting developments in the field. ${ }^{3}$ However, the deactivation of heterogeneous catalysts is a ubiquitous problem, yet to be resolved. ${ }^{4}$ Particularly in the petroleum industry, many sacrificial oxidative or reductive processes are utilized in order to extend the catalytic activity, and in the process to decrease the quantities of spent petroleum catalysts. ${ }^{5}$ Our contribution deals with smaller scale production, as it may be applicable to pharmaceuticals, fine chemicals and high value added organic synthesis. We have previously reported on Pddecorated $\mathrm{TiO}_{2},{ }^{6-8}$ a versatile material that can be used for different organic transformations, showing variable catalyst longevity. In order to develop sustainable recovery strategies, we propose a strategy based on a reaction rotation methodology, a method we label catalytic farming due to its resemblance to agricultural practices. ${ }^{9,10}$ As it is well-known in agriculture, the

Department of Chemistry and Biomolecular Sciences, Centre for Advanced Materials Research (CAMaR), University of Ottawa, 10 Marie Curie, Ottawa, Ontario K1N 6N5, Canada.E-mail:jscaiano@uottawa.ca; alantern@uottawa.ca

$\dagger$ Electronic supplementary information (ESI) available: TEM catalyst characterization, graphs of conversions and yields and multiple product tables for several reaction rotations. See DOI: $10.1039 / \mathrm{c} 8 \mathrm{sc} 04188 \mathrm{a}$ continuous use of the same crop can reduce the content of certain nutrients in the soil, thus reducing the soil yield. With crop rotation, farmers ensure that the nutrients leached or restored by one crop can be absorbed by the following one in the next growing season. The same idea can be extrapolated to catalysis: one reaction can deplete the catalyst of certain properties that can be restored by another reaction during the next catalytic cycle. The ability to reuse catalysts is consistent with the principles of green chemistry, which encourages the use of sustainable practices. ${ }^{11}$

\section{Results and discussion}

In this work, we demonstrate that rotating the catalytic processes to synthesize alternating target molecules achieves these goals. Just as retaining the properties and richness of the soil is key to agriculture, having a robust catalyst able to perform many catalytic cycles is essential for their efficient and prolonged application in organic synthesis.

Our choice of catalyst to illustrate our rotation strategy is palladium nanoparticles $(\sim 1.3 \mathrm{~nm}, 2$ wt\%) deposited on nanometric $\mathrm{TiO}_{2}\left(\mathrm{Pd} @ \mathrm{TiO}_{2}\right)$, predominantly in its anatase form, a catalyst that we have utilized for several organic transformations, ${ }^{6-8}$ including $\mathrm{C}-\mathrm{C}$ coupling reactions (Ullmann homo-coupling and Sonogashira coupling). ${ }^{6,7}$ These are among the examples we use to illustrate the catalytic farming concept, along with alkene isomerization (or hydrogenation) ${ }^{8}$ also described in this contribution (Scheme 1). We based our reaction selection on previous mechanistic studies that include the role of the solvent and evaluation of the catalyst after each reaction. Thus, while catalytic Sonogashira reaction deteriorates rapidly with usage (problem reaction), catalytic Ullmann 

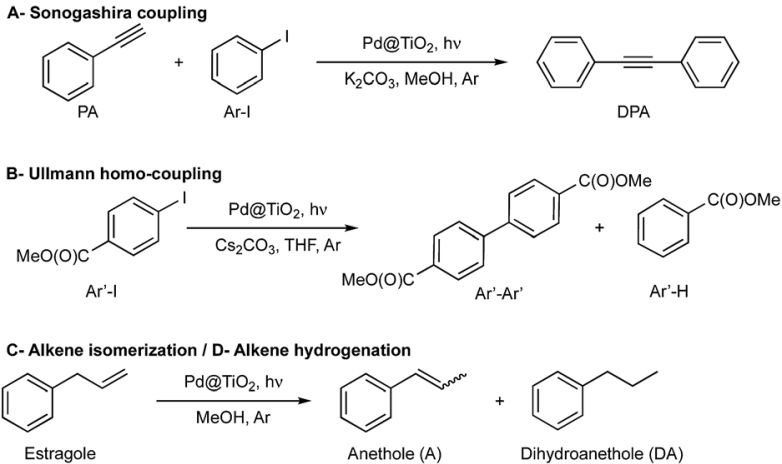

Scheme 1 Reactions used to demonstrate the catalytic farming concept. (A) Sonogashira coupling ${ }^{7}$ is catalyzed by supported PdNP upon visible light irradiation in methanol $(\mathrm{MeOH})$ and $\mathrm{Ar}$ atmosphere in the presence of base $\left(\mathrm{K}_{2} \mathrm{CO}_{3}\right)$. (B) Ullmann homo-coupling of methyl 4-iodobenzoate $\left(\mathrm{Ar}^{\prime}-\mathrm{I}\right)$ proceeds under UV-vis light irradiation in the presence of catalyst $\left(\mathrm{Pd}\left(\mathrm{CTiO}_{2}\right)\right.$ and base $\left(\mathrm{Cs}_{2} \mathrm{CO}_{3}\right)$ utilizing tetrahydrofuran (THF) as solvent and Ar atmosphere. (C) Alkene isomerization $^{8}$ of estragole can be carried out upon blue light irradiation of a methanolic suspension of $\mathrm{PdaTiO}_{2}$ under argon atmosphere. (D) Alkene hydrogenation of estragole can be performed under the isomerization conditions by switching the light to UV light.

reaction shows great catalyst recyclability and is a plausible recovery reaction; as illustrated in Fig. 1 . Note that we use the word 'deterioration' rather than the more conventional 'poisoning'; the latter suggests contamination by some unwanted material, while deterioration seems a broader description, perhaps more suitable in this case where the results suggest that changes in the oxidation state of palladium (vide infra) may be behind the reduced catalyst performance.

Inspired by the crop rotation process utilized in agriculture, we realized the importance of understanding the reaction mechanism as well as the properties of the catalytic material used. Mechanistically the reactions of Scheme 1 involve electron-hole charge separation when irradiated in the UVA region and direct excitation of the Pd nanoparticles when visible light is used (Scheme 2). Further details appear in earlier publications. ${ }^{6-8}$ It is important to highlight that the solvents used for
A: Sonogashira coupling

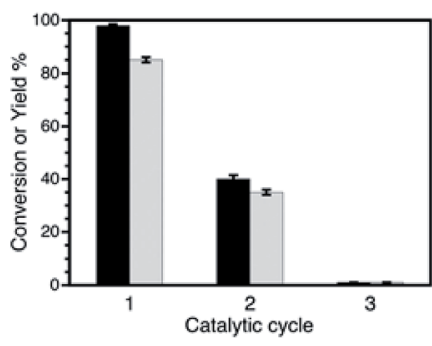

Fig. 1 Conversions (dark bars) and yields (light bars) obtained after several catalytic cycles of reactions (A) and (B) in Scheme 1. While reaction $(A)$ experiences a dramatic efficiency drop, reaction (B) can be catalyzed with excellent conversions and yields for several catalytic cycles. Reaction conditions: (A) Sonogashira coupling upon $450 \mathrm{~nm}$ irradiation at $2.7 \mathrm{~W} \mathrm{~cm}^{-2}$, (B) Ullmann homo-coupling upon $368 \mathrm{~nm}$ and $465 \mathrm{~nm}$ irradiation at 0.3 and $1.6 \mathrm{~W} \mathrm{~cm}^{-2}$, respectively.
(A)

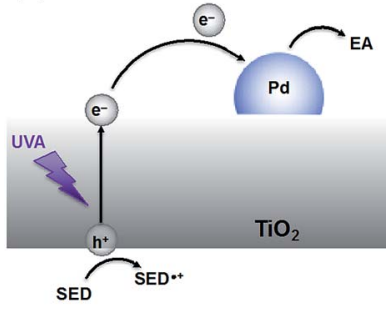

(B)

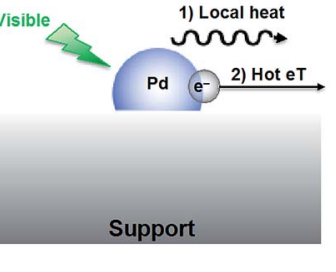

!

Scheme 2 Suggested mechanisms under UVA (A) or visible (B) irradiation. (A) Upon UVA excitation an electron is pumped from the valence band (VB) into the conduction band (CB) of the semiconductor $\left(\mathrm{TiO}_{2}\right)$. The electron can be trapped by the Pd nanoparticle attached to the surface slowing down the electron-hole recombination kinetics. Therefore, electron acceptor reagents (EA) can react more easily on the catalyst surface whereas a sacrificial electron donor (SED), frequently the solvent, quenches the hole. (B) Under visible light excitation, the generation of hot electrons on the Pd surface can photocatalyze reactions through (1) local heat generation or (2) hot electron transfer (eT), ${ }^{12}$ the latter being the accepted mechanism for this type of non-plasmonic nanoparticles.

each reaction play an important role on the activity and reusability of this catalyst, actively participating in the reaction mechanism.

A closer look to Sonogashira reaction has shown ${ }^{7}$ that the decreased catalytic performance indicated in reaction $\mathrm{A}$ (Scheme 1) is not due to the leaching of Pd species from $\mathrm{Pd} @ \mathrm{TiO}_{2}$; in fact, ICP measurements revealed that $>97 \%$ of the $\mathrm{Pd}$ is retained by the catalyst after three reaction cycles. Further, the reaction supernatant shows no catalytic activity after separation from the solid catalyst, reinforcing the heterogeneous nature of the reaction. ${ }^{7}$ Material characterization showed that particle size is not altered during the catalytic processes.

With all this in mind, we decided to perform XPS analyses of the materials. One can observe that the HR-XPS spectrum of Pd changes after the first cycle of Sonogashira reaction. These changes, illustrated in Fig. 2A and B are consistent with changes in the oxidation state of Pd on the catalyst surface. For instance, XPS analysis of the fresh catalyst suggests the presence of $\mathrm{PdO},{ }^{8}$ with a small contribution of more reduced palladium species. In contrast, more reduced Pd species are found after the first use of the catalyst for the Sonogashira reaction. This change accompanies the loss of activity after the first catalytic cycle. Interestingly, after performing the Ullmann reaction there is less contribution of the reduced Pd species, with less dramatic changes comparing to the fresh material (Fig. 2C), consistent with the great reusability for this reaction. To our surprise, photochemical treatment with THF - the solvent used during Ullmann coupling - restores the oxidation state of Pd to almost the same as in the fresh material, Fig. 2D. This is in agreement with our previous studies over thermal alkene isomerizations ${ }^{8}$ utilizing the same catalyst, where we reported the oxidation of Pd species during the catalytic reaction. We then described photocatalytic treatments performed over used $\mathrm{Pd@TiO}$ catalyst with complete recovery of the catalyst efficiency., ${ }^{7,8}$ Thus, oxidation changes of the Pd surface provide a plausible rationalization for catalyst deterioration, reductive treatments - 


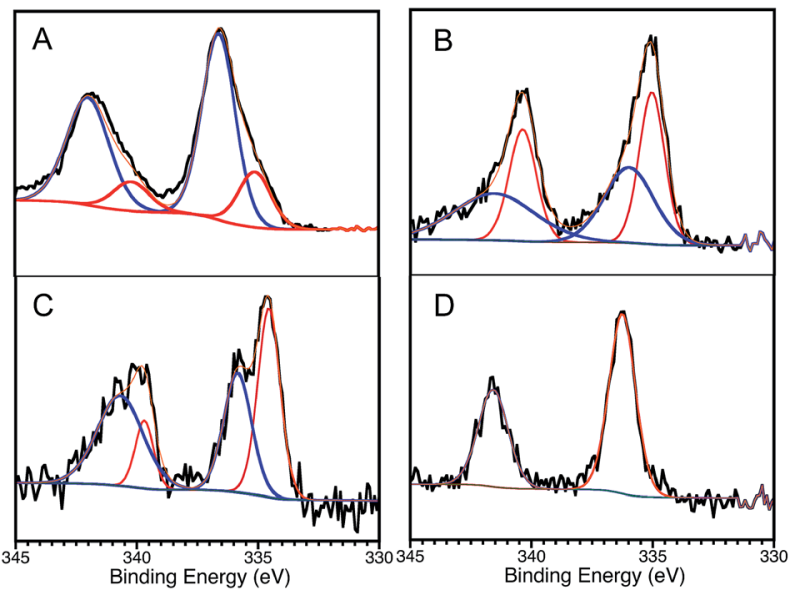

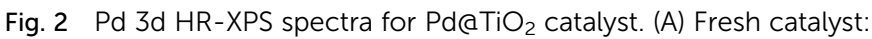
$\mathrm{Pd} 3 \mathrm{~d}$ core-level spectrum deconvoluted by using two spin-orbit split $\mathrm{Pd} 3 \mathrm{~d}_{5 / 2}$ and $\mathrm{Pd} 3 \mathrm{~d}_{3 / 2}$ components centred at $336.6 \mathrm{eV}$ and $342.0 \mathrm{eV}$ and separated by $\sim 5.4 \mathrm{eV}$; attributed to $\mathrm{PdO}^{8}$ Small contribution of more reduced palladium species are also found on the material (components at $335.1 \mathrm{eV}$ and $340.2 \mathrm{eV}$ ). (B) Catalyst after Sonogashira reaction: high contribution of more reduced species (spin-orbit components at 335.0 (336.0) eV and 340.4 (341.5) eV). (C) Catalyst after Ullmann reaction: similar contribution of both oxidized and less oxidized species. (D) Catalyst after Sonogashira reaction and posttreatment with THF: oxidation state of Pd restored to almost the same as in the fresh material (336.1 and $341.5 \mathrm{eV}$ ).

such as irradiation in the presence of a reductive benzoin photoinitiator (I-2959) - completely recovered the catalytic activity. ${ }^{8}$

Considering these observations, we designed a series of different reaction rotations where one would expect the most efficient reactions (Ullmann $\mathrm{C}-\mathrm{C}$ coupling) would help to improve the efficiencies of the poor ones (Sonogashira $\mathrm{C}-\mathrm{C}$ coupling). After screening the reaction-rotation conditions similar to what happens in agriculture - we found the right combination of reactions. Accordingly, rotation with Ullmann reaction remarkably improves the efficiency of the catalyst towards Sonogashira coupling (Fig. 3 ), with up to $80 \%$ yield ( $1 \mathrm{~h}$ ) after 6 catalytic cycles, ESI Table S1. $\dagger$ Clearly the Ullmann reaction assists in maintaining the catalyst performance in the Sonogashira reaction. In the same series of experiments (see ESI Table S2 $\dagger$ ) we show that it is not essential to always alternate the reactions, and that two of the same kind can be performed in sequence.

We were also able to establish that the catalytic farming strategy can be further expanded to additional reactions. Thus, reactions, such as alkene isomerization or hydrogenation, Scheme $1,{ }^{8}$ can contribute to the catalytic farming strategy while retaining catalyst performance. Accordingly, reaction (C) (or (D)) when run independently, can be catalyzed with excellent conversions and yields for several catalytic cycles (Fig. 4).

The addition of the isomerization reaction serves to illustrate the robustness of the reaction rotation strategy. While alkene isomerization does not restore the catalytic activity toward Sonogashira reaction, its inclusion in the catalytic farming process does not alter the performance of Sonogashira $\mathrm{C}-\mathrm{C}$

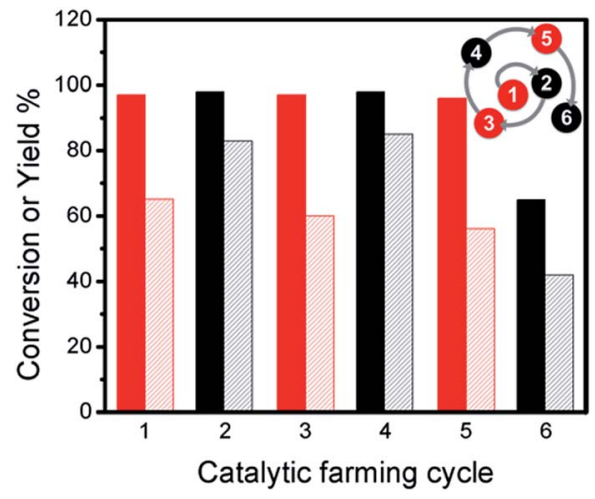

Fig. 3 Conversions (dark bars) and yields (light bars) for catalytic farming of $\mathrm{Pd} \mathrm{aTiO}_{2}$ by rotation between Sonogashira coupling (black) upon $450 \mathrm{~nm}$ irradiation at $2.7 \mathrm{~W} \mathrm{~cm}^{-2}$ for $30 \mathrm{~min}$ and Ullmann homocoupling (red) upon $368 \mathrm{~nm}$ and $465 \mathrm{~nm}$ irradiation at 0.3 and $1.6 \mathrm{~W}$ $\mathrm{cm}^{-2}$ for $1 \mathrm{~h}$. Compare to Fig. $1 \mathrm{~A}$ and $\mathrm{B}$. The spiral at the top-right corner helps us visualize the sequence of reactions with the number representing the reaction sequence, and the color the type of reaction. Similar spirals are included in other figures.

coupling (Fig. 5). In the cases of Fig. 5 it is clear that the Ullmann reaction plays an important role in extending the catalyst lifetime. As already noted, alkene isomerization deactivates the catalyst towards Sonogashira coupling, Fig. 5A, whereas Sonogashira coupling partially retains the catalyst activity towards alkene isomerization. Hence, in the crop rotation analogy, Sonogashira coupling cannot follow isomerization. Likewise, the alkene isomerization is drastically decreased if used as an in-between reaction (Fig. 5 B), however this reaction does not affect the performance of Ullmann as a subsequent reaction (Fig. 5C). Further, Sonogashira coupling shows excellent reactivity in catalytic cycles $4^{\text {th }}$ and $5^{\text {th }}$ with excellent TON numbers (2037 and 2011 TON per Pd NP, respectively - see ESI Table S3 $\dagger$ ). Notice that the rotation outcome also depends on the irradiation conditions; hence, when using lower irradiation intensities, Sonogashira coupling is only partially deactivated by the alkene isomerization reaction (see ESI Table S4†). Similar results are found when using alkene hydrogenation reaction (see ESI Table S5†). Notice that under these conditions after 6 catalytic cycles, the Ullmann reaction no longer serves as
C: Alkene isomerization

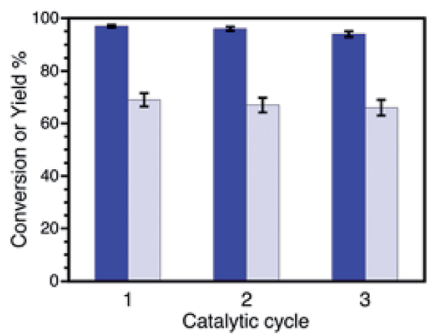

D: Alkene hydrogenation

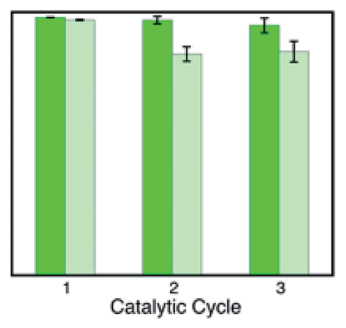

Fig. 4 Conversions (dark bars) and yields (light bars) obtained after several catalytic cycles of (C) alkene isomerization upon $450 \mathrm{~nm}$ irradiation at $2.7 \mathrm{~W} \mathrm{~cm}^{-2}$ and (D) alkene hydrogenation upon $368 \mathrm{~nm}$ irradiation at $0.3 \mathrm{~W} \mathrm{~cm}^{-2}$. Notice that each catalytic cycle implies catalyst separation - cleaning cycles - before reusability test. 


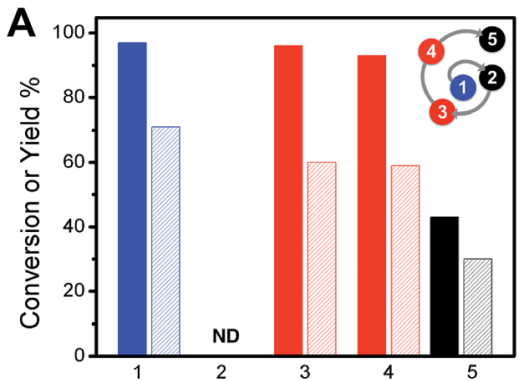

B

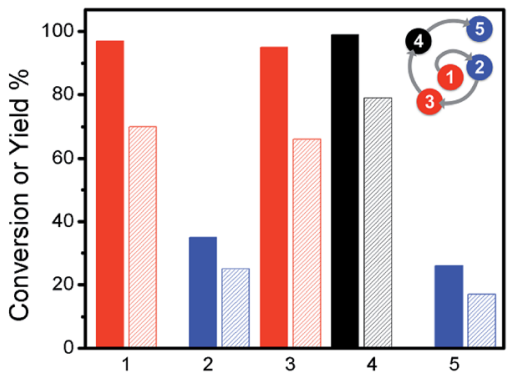

Catalytic farming cycle

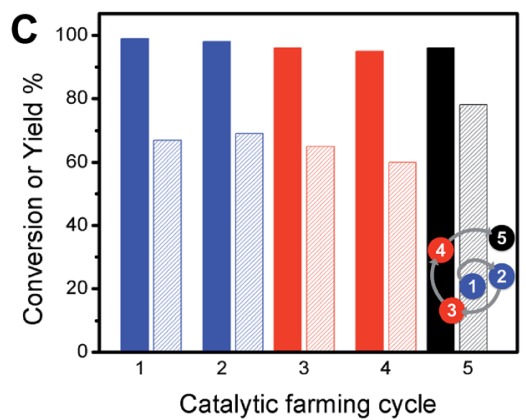

Fig. 5 Conversions (dark bars) and yields (light bars) for catalytic farming of $\mathrm{PdCTiO}_{2}$ by rotation of three different reactions: alkene isomerization (blue), Ullmann homo-coupling (red) and Sonogashira coupling (black). Alternating reactions rotations show different performance for each reaction. See ESI Table S3.†

a "catalyst recovery treatment" and only THF treatment (vide infra) partially restores the Sonogashira reaction (see ESI Table S6 $\dagger$ ).

A summary of the improvements achieved toward Sonogashira coupling using this reaction rotation strategy can be found in Table 1 . The concept of reaction rotation represents a paradigm shift in heterogeneous catalysis by bringing catalyst reuse and recovery in the area of fine chemicals much closer to the principles of green chemistry. In our case, the Ullmann reaction seems to play a critical role in retaining catalyst performance. However, if the Ullmann reaction does not result in desirable or value-added products, then it could be perceived as a sacrificial reaction with potential financial and environmental costs. This brings us back to the practices of organic farming, where similar scenarios can be encountered. In agriculture in some cycles of crop rotation, a "cover crop" is cultivated with the sole objective of protecting and enriching the soil; a typical example is clover. ${ }^{\mathbf{1 3}}$ Similar practices can be implemented in catalytic farming, for example $\mathrm{Pd@TiO}{ }_{2}$ can be irradiated with UVA-blue light in THF, converting some of the
Table 1 Improvement of the catalytic activity of $\mathrm{PdaTiO}$ toward Sonogashira coupling after different reactions rotation ${ }^{a}$

\begin{tabular}{|c|c|c|c|c|c|}
\hline Rotation & Cycle & $\begin{array}{l}\% \\
\text { Yield }\end{array}$ & Rotation & Cycle & $\begin{array}{l}\% \\
\text { Yield }\end{array}$ \\
\hline & 2 & 39 & & 7 & 62 \\
\hline & 3 & 21 & & 5 & 30 \\
\hline & 3 & 25 & & 4 & 79 \\
\hline & 6 & 80 & & 5 & 79 \\
\hline
\end{tabular}

${ }^{a}$ Alternating reactions rotations show different performance for each reaction. Sonogashira coupling (black), Ullmann homo-coupling (red), alkene isomerization (blue) and alkene hydrogenation (green). See Experimental section for reactions conditions.

THF to dihydrofuran and hydrogen and in the process restoring the catalyst (Fig. 6); clearly the same is achieved with the Ullmann reaction (Scheme 1). The choice between Ullmann chemistry or a recovery treatment (the "cover crop" in agriculture) would ultimately be made on the basis of market demands and business practicality, parameters that should be considered on a case-by-case basis. Thus, there is no need of a sacrificial cycle if one step in the rotation offers limited or no benefit.

In summary, we propose a paradigm shift that calls for reaction rotation in heterogeneous catalysis as a strategy to enhance catalyst longevity. We define this practice (catalytic farming) in the following terms: target product rotation is the practice of performing a series of dissimilar or different types of catalytic processes using the same catalyst in sequenced reactions. It is done so that the catalysts are not deactivated by

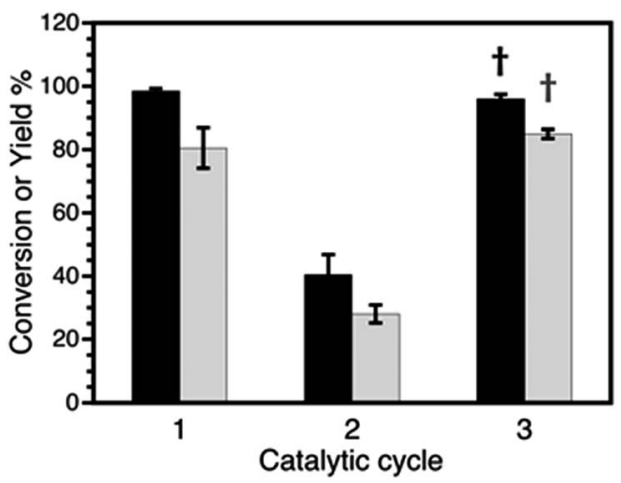

Fig. 6 Conversions (black) and yields (grey) obtained after 3 catalytic cycles for Sonogashira coupling. Cycle 3 shows the recovery of catalytic activity after THF treatment $(\dagger)$. Compare with cycle 3 in Fig. $1 \mathrm{~A}$ and ESI Table S10.† 
a fixed set of reactants. It helps in reducing catalyst deterioration and increases longevity and product yield.

The set of reactions, catalyst, and rotation sequence will depend on the synthetic goals of a given laboratory or organization. Once again, the analogy with agriculture is very enlightening. When 16 farmers in one region were asked to provide their favorite crop rotation strategy, they provided 16 different answers, where some crop rotations make frequent appearances (rye, alfalfa, garlic). ${ }^{14}$ Just as in chemistry, there is no perfect rotation sequence, as external parameters must be considered, including the usefulness of the crop or target product, and in our case, consideration that one reaction may play a key role in maintaining catalyst ("soil") performance. Therefore, different laboratories may create rotation sequences that meet their needs, involving some reactions included mainly for catalyst reactivation, such as the catalyst irradiation in THF in the case above.

\section{Experimental section}

\section{Materials}

Unless otherwise specified, all chemicals were purchased from Sigma-Aldrich or Fisher Scientific and used without further purification. Titanium dioxide $\left(\mathrm{TiO}_{2}-\mathrm{P} 25\right)$ was purchased from Univar Canada. All solvents were of HPLC grade.

\section{Instrumentation}

Transmission electron microscopy (TEM) images were collected on a JEM-2100F FETEM (JEOL) operating at $200 \mathrm{kV}$. The Pd content of the catalysts was determined by Inductively Coupled Plasma Optical Emission Spectrometry (ICP-OES), using Agilent 5110 ICP-OES instrument. Approximately $10 \mathrm{mg}$ portions were accurately weighed in triplicate and digested with aqua regia. Solutions were further diluted and measured by ICP-OES. The Pd $340.458 \mathrm{~nm}$ emission line was used for quantification. The XPS spectra were measured on a Kratos Nova AXIS spectrometer equipped with an Al X-ray source. The XPS data were collected using AlK $\alpha$ radiation at $1486.69 \mathrm{eV}(150 \mathrm{~W}, 15 \mathrm{kV})$, charge neutralizer and a delay-line detector (DLD) consisting of three multi-channel plates. Binding energies are referred to the $\mathrm{C} 1 \mathrm{~s}$ peak at $284.8 \mathrm{eV}$. XPS data was analyzed using CasaXPS software, Version 2.3.15 and all fittings obtained using a Gaussian $30 \%$ Laurentian and a Shirley baseline. UV irradiation used for catalyst synthesis was performed in a Luzchem photoreactor equipped with UVA lamps (typically operated with 14 lamps, corresponding to $\sim 0.029 \mathrm{~W} \mathrm{~cm}^{-2}$ with $\sim 4 \%$ spectral contamination. Light-emitting diodes (centered at 368 and $465 \mathrm{~nm}$, respectively) of $10 \mathrm{~W}$ from LedEngin and Luzchem LED illuminator (LEDi) equipped with a head of seven powerful blue LEDs (centered at $450 \mathrm{~nm}$ ) with adjustable intensity at focal point were used as described for the photocatalytic reactions studied. Quantification was carried out in a Perkin Elmer, Claurus Gas Chromatograph coupled to a Flame Ionization Detector (FID) and a DB-5 column (30 m length, $0.320 \mathrm{~mm}$ diameter, $0.25 \mu \mathrm{m}$ film) using Ar as a carrier gas and $t$-Butyl benzene as external standard. GC-MS analyses were performed on an Agilent 6890-N Gas Chromatograph with an Agilent 5973 mass selective detector calibrated with acetophenone.

\section{Catalyst synthesis}

Palladium nanoparticles $(\sim 2 \quad$ wt $\%)$ supported on $\mathrm{TiO}_{2}$ $\left(\mathrm{Pd} @ \mathrm{TiO}_{2}\right)$, were prepared by photodeposition of PdNP onto $\mathrm{TiO}_{2}$ (P25) and fully characterized as described in our previous report. $^{8}$

\section{Sonogashira C-C coupling}

Visible light-induced Sonogashira C-C coupling was performed as described in our previous report. ${ }^{7}$ In brief, $15 \mathrm{mg}$ of $\mathrm{Pd@(TiO}$ were dispersed in $4 \mathrm{~mL}$ of HPLC grade methanol (MeOH) in a $10 \mathrm{~mL}$ clean tube, then $15 \mu \mathrm{L}$ of iodobenzene (1 eq., 0.13 $\mathrm{mmol}$ ), $18 \mu \mathrm{L}$ of phenylacetylene (1.3 eq., $0.16 \mathrm{mmol}$ ) and $35 \mathrm{mg}$ of $\mathrm{K}_{2} \mathrm{CO}_{3}$ ( 2 eq., $0.26 \mathrm{mmol}$ ), were added. The reaction mixture was purged with Ar for 15 min then irradiated with $1 \times 465 \mathrm{~nm}$ LED set up at $1.6 \mathrm{~W} \mathrm{~cm}^{-2}$ for $5 \mathrm{~h}$ (or $7 \times 450 \mathrm{~nm}$ LEDs set up at $2.7 \mathrm{~W} \mathrm{~cm}^{-2}$ for $30 \mathrm{~min}$ ) under continuous stirring. The solid catalyst was separated by centrifugation. Quantification was done by GC-FID using $t$-butyl benzene as an external standard (see ESI Table S7†).

\section{Alkene isomerization/hydrogenation}

Visible light-induced isomerization (or hydrogenation) of estragole was performed with slight modifications to our previous report. ${ }^{7}$ In brief, $15 \mathrm{mg}$ of $\mathrm{Pd} @ \mathrm{TiO}_{2}$ were dispersed in $4 \mathrm{~mL}$ of HPLC grade $\mathrm{MeOH}$ in a clean quartz cuvette, then $25 \mu \mathrm{L}$ $(0.16 \mathrm{mmol})$ of estragole were added. The reaction mixture was purged with Ar for $15 \mathrm{~min}$ and then irradiated with $7 \times 450 \mathrm{~nm}$ LEDs set up at $2.7 \mathrm{~W} \mathrm{~cm}^{-2}$ for $5 \mathrm{~h}$ under continuous stirring (or with $1 \times 368 \mathrm{~nm}$ LED set up at $0.3 \mathrm{~W} \mathrm{~cm}^{-2}$ for hydrogenation). The progress of the reaction was monitored by GC-MS. The quantification was done by GC-FID using $t$-butyl benzene as an external standard (see ESI Table S8†).

\section{Ullmann homo-coupling}

Light-induced Ullmann homo-coupling of methyl 4-iodobenzoate was carried out based on our recent publication: ${ }^{6}$ $20 \mathrm{mg}$ Pd@ $\mathrm{TiO}_{2}$ were dispersed in $4 \mathrm{~mL}$ of tetrahydrofuran (THF) in a clean quartz tube, then $26 \mathrm{mg}$ ( $0.1 \mathrm{mmol}, 1 \mathrm{eq}$.) of methyl-4 iodobenzoate and $65 \mathrm{mg}\left(0.2 \mathrm{mmol}, 2 \mathrm{eq}\right.$.) of $\mathrm{Cs}_{2} \mathrm{CO}_{3}$ were added. The reaction mixture was purged with Ar for $10 \mathrm{~min}$ prior to irradiation. Irradiation sources used: $1 \times 465 \mathrm{~nm}$ LED plus $1 \times 368 \mathrm{~nm}$ LED set up at 1.6 and $0.3 \mathrm{~W} \mathrm{~cm}^{-2}$, respectively; or $1 \times 368 \mathrm{~nm}$ LED set up at $0.3 \mathrm{~W} \mathrm{~cm}^{-2}$. The progress of the reaction and the quantification were done by GC-FID using $t$ butyl benzene as an external standard (see ESI Table S9†).

\section{Catalyst recyclability}

The catalyst was recovered after each cycle by centrifugation (3500 rpm for $15 \mathrm{~min}$ ). Once the supernatant was decanted, the catalyst was washed three times with $\sim 6 \mathrm{~mL}$ fresh methanol. Each time the catalyst was dispersed via sonication and isolated through centrifugation. The recovered clean catalyst was 
suspended in $4 \mathrm{~mL}$ HPLC grade $\mathrm{MeOH}$ in a clean tube prior to reuse. Reactant concentrations and irradiation time were kept constant for all the cycles and catalyst losses during the recovery process were considered negligible.

\section{Catalyst recovery treatment}

The recovered catalyst from two subsequent cycles of lightinduced Sonogashira reaction was separated from the reaction mixture by centrifugation (3500 rpm for $15 \mathrm{~min}$ ). In the recovery process the catalyst was washed 2 times with $\sim 5 \mathrm{~mL}$ fresh $\mathrm{MeOH}$ then 2 more times with $\sim 5 \mathrm{~mL}$ fresh THF prior to activation. In brief, the recovered $\mathrm{Pd@TiO}{ }_{2}$ was added to $4 \mathrm{~mL}$ THF and $60 \mathrm{mg} \mathrm{Cs}_{2} \mathrm{CO}_{3}$ into a clean quartz tube, suspended by sonication for $10 \mathrm{~min}$ then purged with Ar for $15 \mathrm{~min}$ prior to $368 / 465 \mathrm{~nm}$ irradiation for $\sim 1 \mathrm{~h}$. The activated solid catalyst was separated by centrifugation, washed with $\mathrm{MeOH}$ three times prior to use for a new cycle for light-induced Sonogashira coupling reaction. Catalyst reactivation using the benzoin photoinitiator (I-2959) was performed as previously reported. ${ }^{\mathbf{8}}$

\section{Catalytic farming}

The reaction was scaled up twice to facilitate catalyst recovery and reuse. The catalyst was recovered after each cycle by centrifugation (3500 rpm for $15 \mathrm{~min}$ ). After each cycle the catalyst was dispersed in fresh clean solvent via sonication and isolated through centrifugation for at least 3 times, and finally dried. Reactions were carried out at given conditions (see figure captions).

\section{Turnover number (TON) and turnover frequency (TOF) calculations $^{15}$}

Considering that each PdNP has at least one catalytic active site, the TON and TOF are calculated related to the moles of PdNPs as follows:

$$
\mathrm{TON}=\frac{\text { moles of reagent } \times \text { yield }}{\text { moles of } \operatorname{Pd~} \mathrm{NP}(n)}
$$

where the moles of PdNPs $(n)$ are calculated as follows:

$$
n=\frac{\text { mass of catalyst } \times \mathrm{wt} \% \mathrm{Pd}}{\mathrm{AW} \times \text { number of } \mathrm{Pd} \text { atoms per } \mathrm{NP}(N)}
$$

and $N$ is the number of Pd atoms per nanoparticle and AW the atomic weight:

$$
N=\frac{\mathrm{Pd} \text { NP volume }}{\text { Pd atomic volume }}
$$

TOF in $\mathrm{h}^{-1}$

$$
\mathrm{TOF}=\frac{\mathrm{TON}}{\text { Time of reaction }(\mathrm{h})}
$$

\section{Conclusions}

In this contribution we propose a new approach to extend catalyst lifetimes based on the crop rotation system used in agriculture rotation practices may become a standard strategy in heterogeneous catalysis, just as they do in organic farming. While this contribution demonstrates the benefits of rotation in catalysis, we also recognize that practical applications in industry will have to meet market demands as a consideration in reaction rotation. Both represent changes in societal attitudes that recognize and celebrate the use of sustainable practices.

\section{Conflicts of interest}

There are no conflicts to declare.

\section{Acknowledgements}

This work was supported by the Natural Sciences and Engineering Research Council of Canada, the Canada Foundation for Innovation, and the Canada Research Chairs Program.

\section{References}

1 A. Biffis, P. Centomo, A. Del Zotto and M. Zeccal, Chem. Rev., 2018, 118, 2249.

2 (a) Q. Liu, M. D. Xu, J. Zhao, Z. Yang, C. Z. Qi, M. F. Zeng, R. Xia, X. Z. Cao and B. Y. Wang, Int. J. Biol. Macromol., 2018, 113, 1308; (b) M. F. Zeng, X. Zhang, L. J. Shao, C. Z. Qi and X. M. Zhang, J. Organomet. Chem., 2012, 704, 29; (c) Z. P. Chen, E. Vorobyeva, S. Mitchell, E. Fako, M. A. Ortuno, N. Lopez, S. M. Collins, P. A. Midgley, S. Richard, G. Vile and J. Perez-Ramirez, Nat. Nanotechnol., 2018, 13, 702.

3 (a) C. M. Friend and B. Xu, Acc. Chem. Res., 2017, 50, 517; (b) W. P. Gao, Z. D. Hood and M. F. Chi, Acc. Chem. Res., 2017, 50, 787; (c) A. Corma and H. Garcia, Chem. Soc. Rev., 2008, 37, 2096; (d) A. Corma, R. Juarez, M. Boronat, F. Sanchez, M. Iglesias and H. Garcia, Chem. Commun., 2011, 47, 1446.

4 M. Argyle and C. Bartholomew, Catalysts, 2015, 5, 145.

5 A. Akcil, F. Veglio, F. Ferella, M. D. Okudan and A. Tuncuk, J. Waste Manage., 2015, 45, 420.

6 N. Marina, A. E. Lanterna and J. C. Scaiano, ACS Catal., 2018, 7593.

7 A. Elhage, A. E. Lanterna and J. C. Scaiano, ACS Sustainable Chem. Eng., 2018, 6, 1717.

8 A. Elhage, A. E. Lanterna and J. C. Scaiano, ACS Catal., 2017, 7, 250.

9 The electronic Rothamsted Documents Archive, http:// www.era.rothamsted.ac.uk, Accessed April, 2018.

10 Crop Rotation on Organic Farms: A Planning Manual ed., ed. C. 1. Mohler and S. E. Johnson, Natural Resource, Agriculture, and Engineering Service, Ithaca, NY, USA, 2009. 
11 P. Anastas and N. Eghbali, Chem. Soc. Rev., 2010, 39, 301.

12 (a) S. Sarina, E. Jaatinen, Q. Xiao, Y. M. Huang, P. Christopher, J. C. Zhao and H. Y. Zhu, Chem. Phys. Lett., 2017, 8, 2526; (b) T. Tana, X. W. Guo, Q. Xiao, Y. M. Huang, S. Sarina, P. Christopher, J. F. Jia, H. S. Wu and H. Y. Zhu, Chem. Commun., 2016, 52, 11567.
13 K. Koehler-Cole, J. R. Brandle, C. A. Francis, C. A. Shapiro, E. E. Blankenship and P. S. Baenziger, Renew. Agr. Food Syst., 2017, 32, 474.

14 I. R. Chongtham, G. Bergkvist, C. A. Watson, E. Sandstrom, J. Bengtsson and I. Oborn, Biol. Agric. Hortic., 2017, 33, 14. 15 G. K. Hodgson and J. C. Scaiano, ACS Catal., 2018, 8, 2914. 